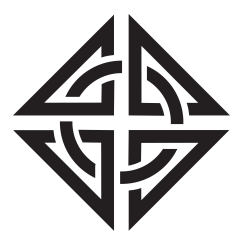

SCIENTIA
Sharif University of Technology

Scientia Iranica

Transactions B: Mechanical Engineering

http://scientiairanica.sharif.edu

\title{
Effects of viscous dissipation and convective heating on convection flow of a second-grade liquid over a stretching surface: An analytical and numerical study
}

\author{
M. Bhuvaneswari ${ }^{a}$, S. Eswaramoorthi ${ }^{b}$, S. Sivasankaran ${ }^{a, *}$, S. Rajan ${ }^{\text {c }}$, \\ and A. Saleh Alshomrani ${ }^{a}$ \\ a. Department of Mathematics, King Abdulaziz University, Jeddah 21589, Saudi Arabia. \\ b. Department of Mathematics, Dr.N.G.P. Arts \& Science College, Coimbatore 641048, Tamil Nadu, India. \\ c. Department of Mathematics, Erode Arts \& Science College, Erode 638009, Tamil Nadu, India.
}

Received 17 May 2017; received in revised form 13 December 2017; accepted 9 April 2018

\section{KEYWORDS}

Second-grade fluid; Convective boundary;

Heat generation;

Suction/injection;

Viscous dissipation.

\begin{abstract}
The effects of viscous dissipation and convective boundary condition on the two-dimensional convective flow of a second-grade liquid over a stretchable surface with suction/injection and heat generation were investigated. The governing partial differential equations were reduced into a dimensionless coupled system of nonlinear ordinary differential equations by appropriate similarity transformation. Then, they were solved analytically by Homotopy Analysis Method (HAM) and numerically by fourth-order Runge-Kutta method with shooting technique. The HAM and numerical results of the local skin friction and local Nusselt number were compared for various emerging parameters. It was found that the momentum boundary layer thickness grew by raising the value of the viscoelastic parameter.

(C) 2019 Sharif University of Technology. All rights reserved.
\end{abstract}

\section{Introduction}

The study of boundary layer flow of a viscoelastic liquid over a stretching surface plays a significant role in manufacturing processes including hot rolling, crystal growing, glass blowing, production of synthetic sheets, thermal insulation, thermal control of nuclear reactors, thermal oil recovery, film cooling, design of thrust bearing, and design of radial diffusers. MHD boundary layer flow and energy transfer of a viscoelastic liquid over a stretchy sheet were investigated by Cortell [1]. Several authors investigated the convective flow and energy transfer behavior of viscoelastic liquid with different physical situations, see [2-8]. In nature, heat

*. Corresponding author.

E-mail address: sd.siva@yahoo.com (S. Sivasankaran) transfer is an important process in many technical and industrial applications such as drying, damage of crops because of freezing, food processing, removal of nuclear fuel debris, underground disposal of radioactive waste materials, etc. Because of these applications, several researchers engage with analyzing the heat transfer process in different physical contexts. Some of the important studies that underline such concept can be found in references [9-14].

The problem of free convective flow of a heat generating/absorbing liquid was studied by Chamkha [15]. He found that the thermal boundary layer thickness increased with the rise of the heat generation or absorption parameter. The heat generation effect on boundary layer flow with different fluids has been investigated in some papers [16-21]. Hayat et al. [22] analyzed convective flow of a second-grade liquid over a stretchy wall with convective surface condition. They found that the energy transfer effects were absent when 
Biot number was zero. Few studies, e.g., [23-26], have investigated the boundary layer flow with convective boundary condition.

So far, no attempt has been made to carry out energy transfer analysis on a second-grade liquid over a stretchy surface with viscous dissipation, internal heat generation/absorption, and convective surface condition. Hence, we study this problem both analytically using the homotopy analysis method and numerically using Runge-Kutta method.

\section{Mathematical formulation}

We consider two-dimensional convective boundary layer flow and energy transfer of a second-grade liquid along a stretchy surface. The surface is stretched with a velocity $u_{w}(x)=b x$, where $b$ is a real number. It is presumed that the liquid is heat generated or absorbed internally. Also, viscous dissipation is taken into consideration. We choose $x$-axis along the stretchy surface and $y$-axis normal to the stretching surface. The governing equations for continuity, momentum, and energy can be written in the following form $[2,22]$ :

$$
\begin{aligned}
& \frac{\partial u}{\partial x}+\frac{\partial v}{\partial y}=0 \\
& u \frac{\partial u}{\partial x}+v \frac{\partial u}{\partial y}=\nu \frac{\partial^{2} u}{\partial y^{2}}+\frac{\alpha_{1}}{\rho}\left(\frac{\partial u}{\partial x} \frac{\partial^{2} u}{\partial y^{2}}+u \frac{\partial^{3} u}{\partial x \partial y^{2}}\right. \\
& \left.+\frac{\partial u}{\partial y} \frac{\partial^{2} v}{\partial y^{2}}+v \frac{\partial^{3} u}{\partial y^{3}}\right) \\
& u \frac{\partial T}{\partial x}+v \frac{\partial T}{\partial y}=\alpha_{m} \frac{\partial^{2} T}{\partial y^{2}}+\frac{\nu}{c_{p}}\left(\frac{\partial u}{\partial y}\right)^{2} \\
& \quad+\frac{\alpha_{1}}{\rho c_{p}}\left(u \frac{\partial u}{\partial y} \frac{\partial^{2} u}{\partial x \partial y}+v \frac{\partial u}{\partial y} \frac{\partial^{2} u}{\partial y^{2}}\right)+\frac{Q}{\rho c_{p}}\left(T-T_{\infty}\right)
\end{aligned}
$$

where $u$ and $v$ are the components of velocity, $x$ and $y$ are the coordinates, $T$ is temperature, $\rho$ is density of the fluid, $\alpha_{1}$ is the second-grade parameter, $Q$ is the coefficient of internal heat absorption $(<0)$ or generation $(>0)$ of the fluid, $c_{p}$ is specific heat, $\nu$ is kinematic viscosity, and $\alpha_{m}$ is thermal diffusivity.

The velocity and thermal boundary conditions can be expressed as follows:

$$
\begin{aligned}
& u=u_{w}(x)=a x, \quad v=v_{w}, \quad-k \frac{\partial T}{\partial y}=h_{c}\left(T_{f}-T\right), \\
& \text { at } y=0, \\
& u \rightarrow 0, v \rightarrow 0, \quad T \rightarrow T_{\infty}, \\
& \text { as } y \rightarrow \infty,
\end{aligned}
$$

where $a$ is a positive constant, $k$ is thermal conductivity of the fluid, $v_{w}$ is suction velocity $(>0)$ or injection velocity $(<0), h_{c}$ is heat transfer coefficient, and $T_{f}$ is temperature of the hot fluid. We define:

$$
\begin{aligned}
& \eta=\sqrt{\frac{a}{\nu}} y, \quad u=a x f^{\prime}(\eta), \\
& v=-\sqrt{a \nu} f(\eta), \quad \theta=\frac{T-T_{\infty}}{T_{f}-T_{\infty}} .
\end{aligned}
$$

Substituting Eq. (4) into Eqs. (2) and (3), we obtain:

$$
\begin{aligned}
& f^{\prime \prime \prime}+f f^{\prime \prime}-f^{\prime 2}+K\left(2 f^{\prime} f^{\prime \prime \prime}-f^{\prime \prime 2}-f f^{i v}\right)=0, \\
& \theta^{\prime \prime}+\operatorname{Pr} f \theta^{\prime}+\operatorname{PrEc} f^{\prime \prime 2}+\operatorname{PrEc} K\left(f^{\prime} f^{\prime \prime 2}-f f^{\prime \prime} f^{\prime \prime \prime}\right) \\
& \quad+\operatorname{Pr} H g \theta=0,
\end{aligned}
$$

with boundary conditions:

$$
\begin{aligned}
& f(0)=f_{w}, \quad f^{\prime}(0)=\frac{b}{a}=c, \quad \theta^{\prime}(0)=-\operatorname{Bi}[1-\theta(0)], \\
& f^{\prime}(\infty)=0, \quad \theta(\infty)=0,
\end{aligned}
$$

where $K=\frac{\alpha_{1} a}{\mu}$ is viscoelastic parameter, $\operatorname{Pr}=\frac{\nu}{\alpha_{m}}$ is the Prandtl number, $\mathrm{Ec}=\frac{u_{w}^{2}}{c_{p}\left(T_{f}-T_{\infty}\right)}$ is the Eckert number, $H g=\frac{Q}{a \rho c_{p}}$ is heat generation $(>0)$ and absorption $(<0)$ parameter, $f_{w}=-\frac{v_{w}}{\sqrt{a \nu}}$ is suction $(>$ 0 ) and injection $(<0)$ parameter, $c=\frac{b}{a}$ is stretching ratio, $\mathrm{Bi}=\frac{h_{c}}{k}\left(\frac{\nu}{a}\right)^{\frac{1}{2}}$ is Biot number, and $\operatorname{Re}=\frac{u_{w} x}{\nu}$ is local Reynolds number.

The local skin friction coefficient $(C f)$ and the local Nusselt number $(\mathrm{Nu})$ are defined as:

$$
C f=\frac{2 \tau_{w}}{\rho u_{w}^{2}}, \quad \mathrm{Nu}=\frac{x q_{w}}{k\left(T_{f}-T_{\infty}\right)},
$$

where $\tau_{w}$ is wall shear stress and $q_{w}$ is the heat flux, which are given by:

$$
\begin{aligned}
\tau_{w} & =\mu\left(\frac{\partial u}{\partial y}\right)_{y=0}+\frac{\alpha_{1}}{\rho}\left(u \frac{\partial^{2} u}{\partial x \partial y}+v \frac{\partial^{2} v}{\partial y^{2}}-2 \frac{\partial v}{\partial y} \frac{\partial u}{\partial y}\right)_{y=0}, \\
q_{w} & =-k\left(\frac{\partial T}{\partial y}\right)_{y=0} .
\end{aligned}
$$

Accordingly, the dimensionless form of local skin friction coefficient and the local Nusselt number are:

$$
\begin{aligned}
& \frac{1}{2} C f \operatorname{Re}^{\frac{1}{2}}=(1+3 K) f^{\prime \prime}(0), \\
& \mathrm{Nu} / \operatorname{Re}^{\frac{1}{2}}=-\theta^{\prime}(0)
\end{aligned}
$$

\section{Solutions}

\subsection{HAM solution}

The solution to the governing system of ordinary 


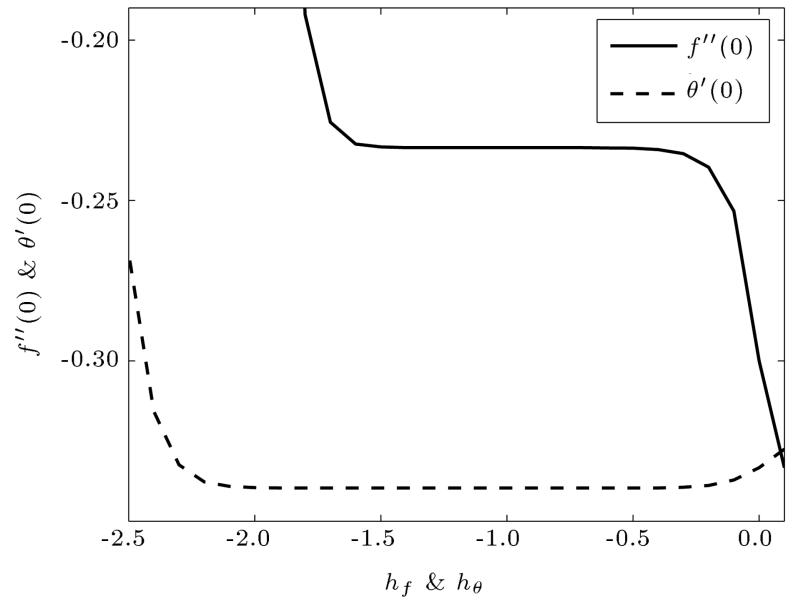

Figure 1. $h$ curves of $f^{\prime \prime}(0)$ and $\theta^{\prime}(0)$ with $K=0.2, c=0.3, f_{w}=0.5, \mathrm{Ec}=0.3, \mathrm{Hg}=-0.5$, and $\mathrm{Bi}=0.5$.

Table 1. Convergence of homotopy analysis solution for different orders of approximation when $K=0.2, c=0.3$, $f_{w}=0.5, \mathrm{Ec}=0.3, \mathrm{Hg}=-0.5$, and $\mathrm{Bi}=0.5$.

\begin{tabular}{ccc}
\hline $\begin{array}{c}\text { Order of } \\
\text { approximation }\end{array}$ & $-\boldsymbol{f}^{\prime \prime}(\mathbf{0})$ & $-\boldsymbol{\theta}^{\prime}(\mathbf{0})$ \\
\hline 1 & 0.24600 & 0.33541 \\
5 & 0.23397 & 0.33958 \\
10 & 0.23349 & 0.33958 \\
15 & 0.23347 & 0.33958 \\
20 & 0.23347 & 0.33958 \\
25 & 0.23347 & 0.33958 \\
30 & 0.23347 & 0.33958 \\
35 & 0.23347 & 0.33958 \\
\hline
\end{tabular}

differential equations (5) and (6) is obtained by using homotopy analysis method. In HAM, first, we choose the initial approximations as $f_{0}(\eta)=f_{w}+c\left(1-e^{-\eta}\right)$ and $\theta_{0}(\eta)=\frac{\mathrm{Bi}^{-\eta}}{1+\mathrm{Bi}}$. The auxiliary linear operators are $L_{f}=\frac{d^{3} f}{d \eta^{3}}-\frac{d f}{d \eta}$ and $L_{\theta}=\frac{d^{2} \theta}{d \eta^{2}}-\theta$. After substituting the $m$ th order deformation equations, the resulting system is solved using HAM with the 15th order of approximations. All the symbolic computations are carried out by MATLAB.

The solutions strongly depend on the auxiliary parameters $h_{f}$ and $h_{\theta}$. In order to see the rates for admissible values of $h_{f}$ and $h_{\theta}$, the $h$-curves of the functions $f^{\prime \prime}(0)$ and $\theta^{\prime}(0)$ are plotted in Figure 1 . The figure indicates that the ranges of admissible values of $h_{f}$ and $h_{\theta}$ are $-1.4 \leq h_{f} \leq-0.6$ and $-1.8 \leq$ $h_{\theta} \leq-0.5$, respectively. If we choose the values of auxiliary parameter $(h)$ from this range, we acquire more precise results. Further, the series converges in the whole region of $\eta$ when $h_{f}=h_{\theta}=-1.0$. Table 1 presents the order of approximations which are necessary for a convergent solution. It is clear that the 15th order of approximation is sufficient for the velocity and temperature profiles.

\subsection{Numerical solution}

Eqs. (5) and (6) are highly nonlinear ordinary differential equations. It is critical to solve these equations. The resulting system of equations with the boundary conditions ( 7 ) is numerically solved using Runge-Kutta fourth-order method with initial guessing $f^{\prime \prime}(0)$ and $\theta^{\prime}(0)$. This process is repeated until we get the required degree of accuracy. The analytical and numerical results are compared in Tables 2 and 3. They assure our analytical and numerical results.

\section{Results and discussion}

In this section, we present the graphical results for velocity profile $\left(f^{\prime}(\eta)\right)$, temperature profile $(\theta(\eta))$, local skin friction $\left(\frac{1}{2} C_{f} \operatorname{Re}^{\frac{1}{2}}\right)$, and local Nusselt number $\left(\mathrm{Nu} / \mathrm{Re}^{\frac{1}{2}}\right)$ with different values of viscoelastic parameter, Eckert number, suction/injection parameter, heat absorption/generation parameter, stretching ratio, and Biot number and fixed value of Prandtl number $(\mathrm{Pr}=$ 1.0).

Table 2 shows the local skin friction and Nusselt number for various values of $K, c$, and $f_{w}$. It is observable that the local skin friction diminishes on raising the values of $K, c$, and $f_{w}$. It is also clear that the local Nusselt number increases on raising the values of $K$ and $f_{w}$. The heat transfer rate is enhanced for slow stretching $c \leq 0.3$; after this, energy transfer rate diminishes. Table 3 represents the local Nusselt number for various values of $\mathrm{Hg}$ and $\mathrm{Bi}$. It is seen that the local Nusselt number rises by raising the Biot number. The heat transfer rate diminishes in the heat absorption case, because the fluid absorbs the heat and reduces the energy transfer rate along the boundary. The high heat transfer rate in internal heat generation case is visualized for $H g \geq 0.5$.

Figure 2(a) and (b) displays the variations of suction $\left(f_{w}>0\right)$ and injection $\left(f_{w}<0\right)$ parameters in velocity profile. It is seen from these graphs that the fluid velocity decreases on increasing the suction parameter. However, the velocity increases with increase in the injection parameter. Therefore, we can control the boundary layer flow using suction. The suction stabilizes the boundary layer growth and the injection destabilizes the boundary layer growth. Figure 3(a) and (b) shows the effects of heat generation $(H g>0)$ and heat absorption $(H g<0)$ parameters on temperature profile. It is found that the temperature increases with increase in the heat generation parameter. However, temperature is a decreasing function of heat absorption parameter. The outcome of internal heat generation is the rise in the rate of energy 
Table 2. The local skin friction coefficient and the local Nusselt number for different values of $K, c$, and $f_{w}$ when $\mathrm{Ec}=0.3, \mathrm{Hg}=-0.5$, and $\mathrm{Bi}=0.5$.

\begin{tabular}{ccccccccc}
\hline $\boldsymbol{K}$ & $\boldsymbol{c}$ & $\boldsymbol{f}_{\boldsymbol{w}}$ & \multicolumn{7}{c}{$\boldsymbol{C} \boldsymbol{f}$} \\
\cline { 3 - 8 } & & & HAM & Numerical & Error (\%) & HAM & Numerical & Error(\%) \\
\hline 0.0 & 0.3 & 0.5 & -0.255625 & -0.254190 & 0.56 & 0.339481 & 0.338971 & 0.15 \\
0.1 & & & -0.316714 & -0.315082 & 0.52 & 0.339546 & 0.339013 & 0.16 \\
0.2 & & & -0.373553 & -0.372914 & 0.17 & 0.339581 & 0.339158 & 0.12 \\
0.3 & & & -0.426924 & -0.426092 & 0.19 & 0.339595 & 0.339242 & 0.10 \\
\hline 0.2 & 0.0 & 0.5 & 0.000000 & 0.000000 & 0.00 & 0.333333 & 0.333298 & 0.01 \\
& 0.3 & & -0.373553 & -0.372914 & 0.17 & 0.339581 & 0.339158 & 0.12 \\
& 0.5 & & -0.713504 & -0.713011 & 0.07 & 0.337943 & 0.338024 & 0.02 \\
& 1.0 & & -1.737367 & -1.731987 & 0.31 & 0.310850 & 0.310762 & 0.03 \\
& 1.5 & & -2.922988 & -2.922194 & 0.03 & 0.248224 & 0.248067 & 0.06 \\
& 2.0 & & -4.216972 & -4.216525 & 0.01 & 0.150971 & 0.151000 & 0.02 \\
\hline 0.2 & 0.3 & -2.0 & 4.019258 & 4.021158 & 0.05 & 0.133571 & 0.133382 & 0.14 \\
& -1.0 & -0.115475 & -0.115019 & 0.39 & 0.223018 & 0.222957 & 0.03 \\
& -0.5 & -0.167777 & -0.166943 & 0.50 & 0.263387 & 0.263951 & 0.21 \\
& 0.0 & -0.255377 & -0.256191 & 0.32 & 0.303996 & 0.303845 & 0.05 \\
& 0.5 & -0.373553 & -0.372914 & 0.17 & 0.339581 & 0.339158 & 0.12 \\
& 1.0 & -0.490922 & -0.490879 & 0.01 & 0.368094 & 0.367924 & 0.05 \\
& 1.5 & -0.587097 & -0.586917 & 0.03 & 0.389865 & 0.388455 & 0.36 \\
& 2.0 & -0.662446 & -0.661953 & 0.07 & 0.411623 & 0.410958 & 0.16 \\
\hline
\end{tabular}

Table 3. The local Nusselt number for different values of $H g$ and $\mathrm{Bi}$ when $K=0.2, c=0.3, f_{w}=0.5$, and $\mathrm{Ec}=0.3$.

\begin{tabular}{|c|c|c|c|c|}
\hline \multirow[t]{2}{*}{$\boldsymbol{H g}$} & \multirow[t]{2}{*}{$\mathrm{Bi}$} & \multicolumn{3}{|c|}{$\mathrm{Nu}$} \\
\hline & & HAM & Numerical & Error (\%) \\
\hline-1.0 & 0.5 & 0.362380 & 0.361992 & 0.11 \\
\hline-0.7 & & 0.350318 & 0.351846 & 0.44 \\
\hline-0.5 & & 0.339581 & 0.338957 & 0.18 \\
\hline-0.3 & & 0.325061 & 0.325001 & 0.02 \\
\hline 0.0 & & 0.286097 & 0.285952 & 0.05 \\
\hline 0.3 & & 0.101896 & 0.101657 & 0.23 \\
\hline 0.5 & & 0.836841 & 0.835927 & 0.11 \\
\hline 0.7 & & 6.589394 & 6.590428 & 0.02 \\
\hline \multirow[t]{11}{*}{-0.5} & 0.0 & 0.000000 & 0.000000 & 0.00 \\
\hline & 0.1 & 0.090796 & 0.091582 & 0.87 \\
\hline & 0.5 & 0.339581 & 0.338953 & 0.18 \\
\hline & 1.0 & 0.516477 & 0.516090 & 0.07 \\
\hline & 1.5 & 0.625004 & 0.624991 & 0.00 \\
\hline & 2.0 & 0.698378 & 0.697753 & 0.09 \\
\hline & 2.5 & 0.751299 & 0.749852 & 0.19 \\
\hline & 3.0 & 0.791272 & 0.790581 & 0.09 \\
\hline & 4.0 & 0.847646 & 0.846928 & 0.08 \\
\hline & 5.0 & 0.885499 & 0.884398 & 0.12 \\
\hline & 10.0 & 0.972341 & 0.971573 & 0.08 \\
\hline
\end{tabular}

transport to the liquid, thereby raising the temperature of the liquid. However, heat absorption is to diminish the rate of energy transport to the liquid, thereby decreasing the temperature of the liquid. The effect of
Eckert number on temperature profile is displayed in Figure 4. It is determined that the thermal boundary layer thickness increases on increasing the Eckert number. That is, the enthalpy inside the boundary layer increases by raising the values of Ec and this produces thicker thermal boundary layer.

Figure 5 shows the various values of Biot number on temperature profile. The fluid temperature is zero for $\mathrm{Bi}=0$, that is, the heat transfer coefficient is zero here. It is observed that the fluid temperature and thermal boundary layer thickness increase on increasing the Biot number (heat transfer coefficient). Figure 6(a)-(c) show the variation of local skin friction for different values of viscoelastic parameter, stretching ratio parameter, and suction/injection parameter. It is observed that the local skin friction decreases on raising the values $c, f_{w}$, and $K$. The local skin friction slowly decreases when raising the values of $f_{w}$ up to $f_{w}=-0.5$, and it decreases rapidly on increasing $f_{w}$.

Figure $7(\mathrm{a})-(\mathrm{d})$ shows the variation of the local Nusselt number for various combinations of suction/injection, heat generation/absorption, Eckert number, and Biot number. It is observed from Figure $7(\mathrm{a})$ that the surface temperature gradient increases on increasing the Biot number. The increment in Nusselt number with $\mathrm{Bi}$ is higher in the presence of suction than in the presence of injection. The heat transfer rate is almost constant at low Biot number $(\mathrm{Bi}=0)$ on increasing $f_{w}$. However, heat transfer rate increases rapidly on increasing $f_{w}$ for higher values of 


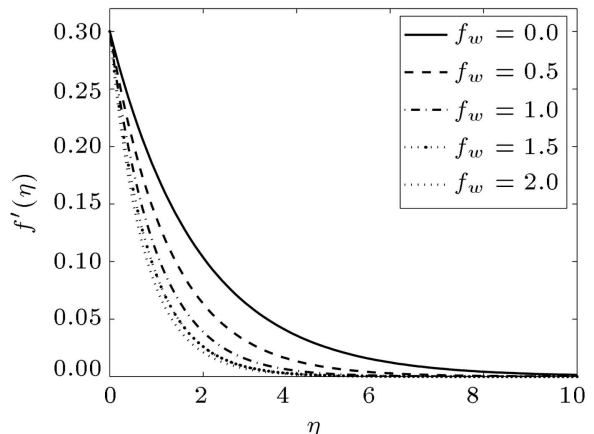

(a)

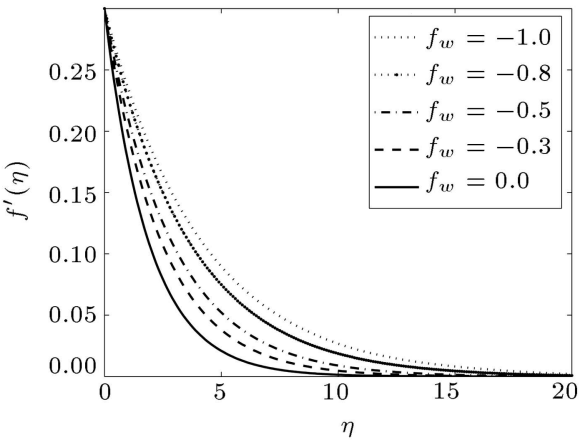

(b)

Figure 2. Velocity profile for different values of (a) suction parameter $\left(f_{w}>0\right)$ and (b) injection parameter $\left(f_{w}<0\right)$; $c=0.3, \mathrm{Ec}=0.3, \mathrm{Hg}=-0.5$, and $\mathrm{Bi}=0.5$.

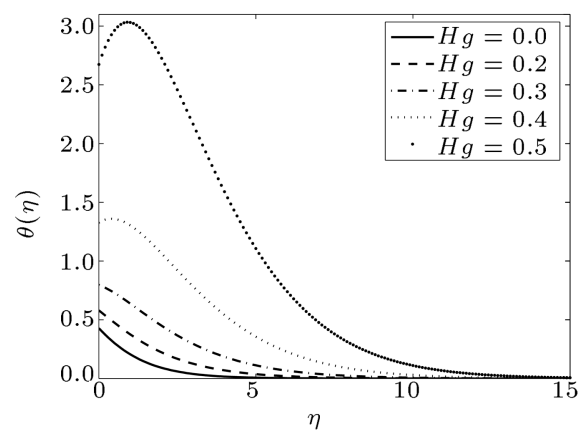

(a)

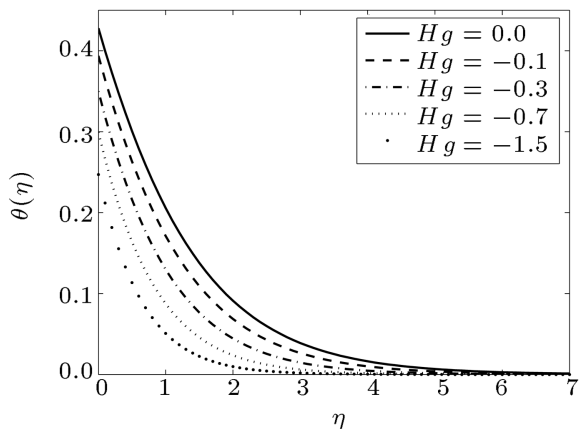

(b)

Figure 3. Temperature profile for different values of (a) heat generation parameter $(H g>0)$ and $(b)$ heat absorption parameter $(H g<0) ; K=0.2, c=0.3, f_{w}=0.5, \mathrm{Ec}=0.3$, and $\mathrm{Bi}=0.5$.

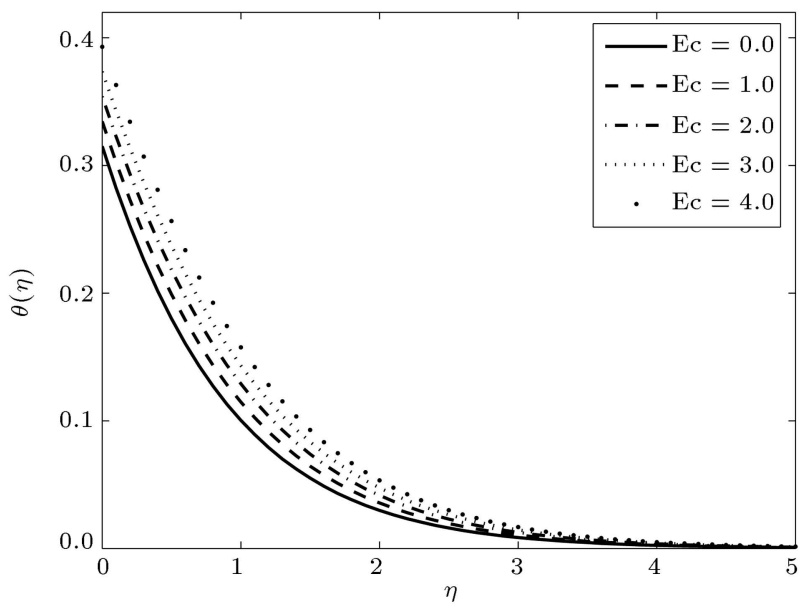

Figure 4. Temperature profile for different values of Eckert number (Ec) with $K=0.2, c=0.3, f_{w}=0.5$, $H g=-0.5$, and $\mathrm{Bi}=0.5$.

Bi. It is seen from Figure $7(\mathrm{~b})$ that the local Nusselt number decreases on raising the values of $\mathrm{Hg}$. This is because surface temperature gradient diminishes on increasing $H g$. The heat transfer rate vanishes when $H g=0.371$. It is found that the critical value of $H g$ is 0.371 . At this point $\left(H g_{\text {crit }}=0.371\right)$, the

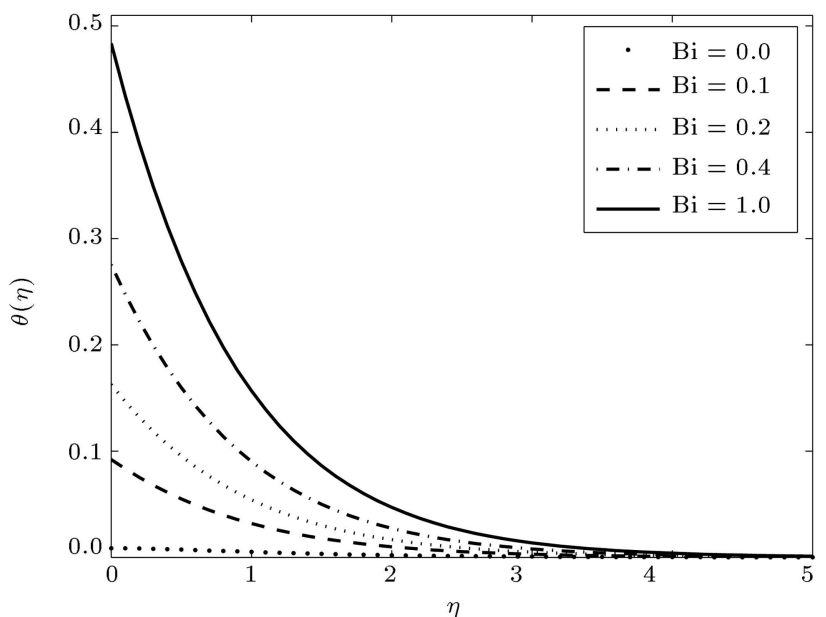

Figure 5. Temperature profile for different values of Biot number (Bi) with $K=0.2, c=0.3, f_{w}=0.5, \mathrm{Ec}=0.3$, and $H g=-0.5$.

local Nusselt number vanishes. Therefore, the internal heat generation rate is equal to surface temperature at $H g_{\text {crit }}=0.371$. Therefore, there is no energy transport between the surface and liquid medium. This is an important result for thermal engineering applications. Before $H g_{\text {crit }}=0.371$, the decrease 


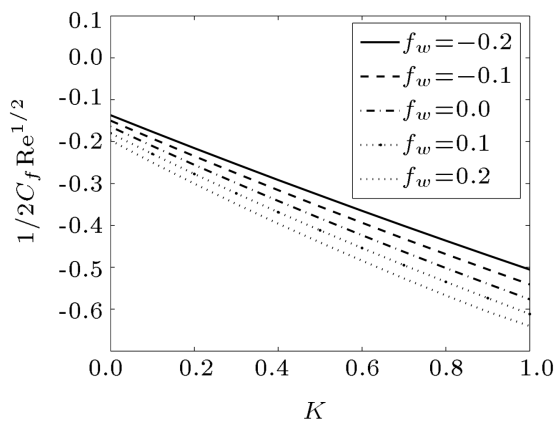

(a)

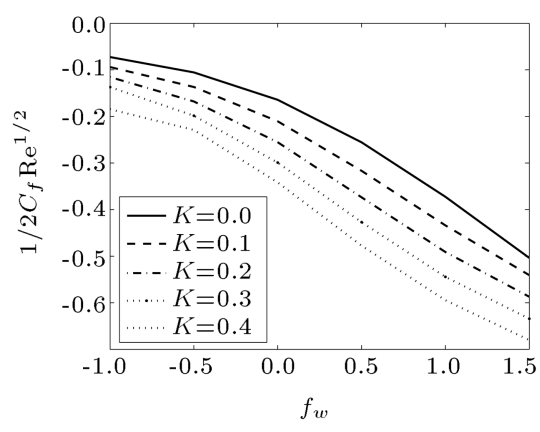

(b)

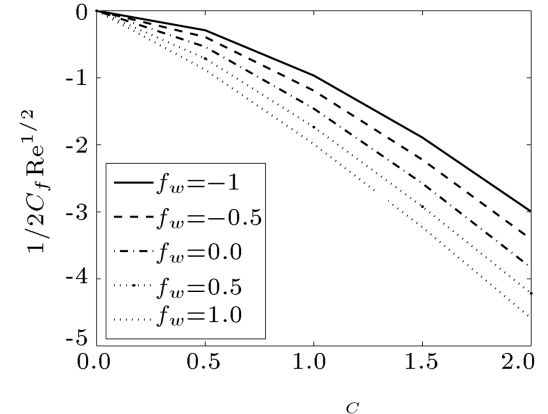

(c)

Figure 6. Variation of the local skin friction for different values of $K, c$, and $f_{w}$.

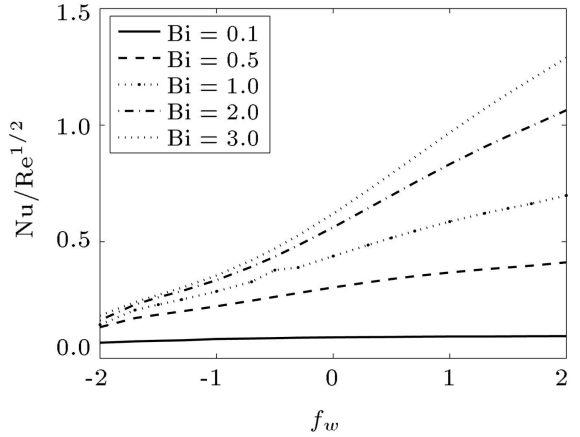

(a)

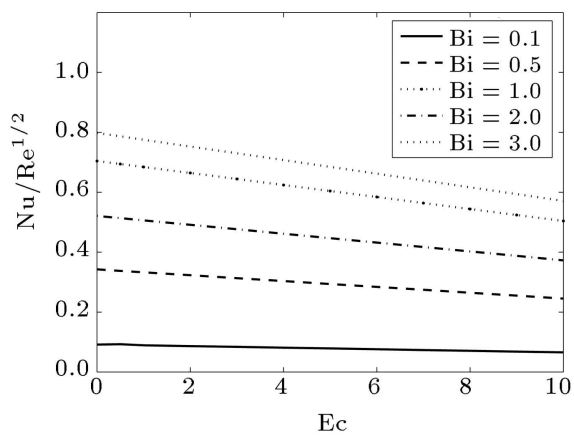

(c)

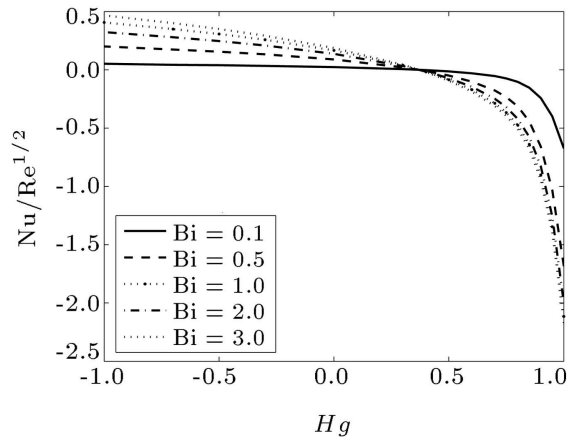

(b)

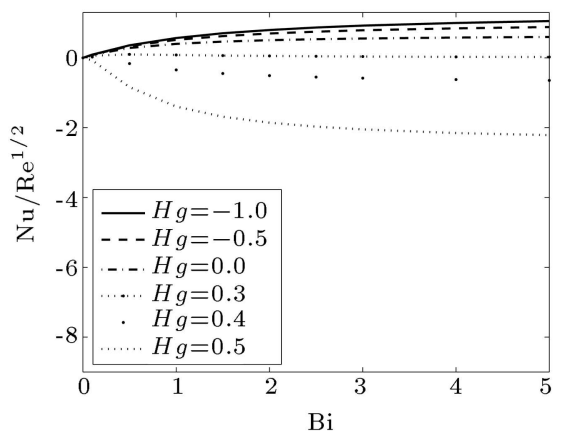

(d)

Figure 7. Variation of the local Nusselt for different values of $f_{w}, \mathrm{Ec}, \mathrm{Hg}$, and Bi with $K=0.2$ and $c=0.3$.

in heat transfer rate is significantly small and after that, it is very high. The Nusselt number decreases exponentially when $\mathrm{Hg}$ approaches unity. The heat transfer rate is reduced slowly on increasing $\mathrm{Hg}$ up to $H g=0.5$. However, after that, heat transfer rate decreases exponentially. It is found from Figure $7(\mathrm{c})$ that the higher values of Eckert number suppress the heat transfer rate. The rate of decrement in Nusselt number with Ec increases for higher values of Biot number. It is concluded from Figure $7(\mathrm{~d})$ that the local Nusselt number decreases on increasing $H g$. In heat absorption case, the local Nusselt number is enhanced with increasing the values of Biot number. The local Nusselt number rapidly decreases with increasing the low values of Biot number for the heat generation case.

\section{Conclusions}

The present study elaborated the effects of viscous dissipation and convective heating on the boundary layer flow and heat transfer of a second-grade liquid. The governing boundary layer systems were transformed into a set of ordinary differential equations with the help of similarity variables. Then, these equations were solved analytically by using homotopy analysis method and numerically by using the Runge-Kutta 4th order method. The following results were concluded from the study:

- The momentum boundary layer thickness was controlled by the suction and it increased by increasing the injection; 
- The thermal boundary layer thickness was enhanced in the presence of heat generation parameter and it decreased when increasing the heat absorption parameter;

- The thermal boundary layer thickness increased with the help of the Eckert and Biot numbers due to the heat dissipation;

- The heat transfer rate increased with increase in the Biot number, and suction/injection parameters, and decreased with increase in the Eckert number and heat generation/absorption parameter.

\section{References}

1. Cortell, R. "Effects of viscous dissipation and work done by deformation on the MHD flow and heat transfer of a viscoelastic fluid over a stretching sheet", shape Physics Letters A, 357(4-5), pp. 298305 (2006).

2. Shawaqfah, M.S., Damseh, R.A., Chamkha, A.J. and Zgoul, M. "Forced convection Blasius flow of secondgrade visco-elastic fluid", Int. J. Heat Tech., 25(1), pp. 145-149 (2007).

3. Abel, M.S. and Mahesha, N. "Heat transfer in MHD viscoelastic fluid flow over a stretching sheet with variable thermal conductivity, non-uniform heat source and radiation", Appl. Math. Model., 32(10), pp. 1965-1983 (2008).

4. Ahmad, A. and Asghar, S. "Flow of a second grade fluid over a sheet stretching with arbitrary velocities subject to a transverse magnetic field", Appl. Math. Letters, 24(11), pp. 1905-1909 (2011).

5. Kecebas, A. and Yurusoy, M. "Numerical solutions of unsteady boundary layer equations for a generalized second grade fluid. J. Theor. Appl. Mech., 49(1), pp. 71-82 (2011).

6. Khan, Y., Hussain, A., and Faraz, N. "Unsteady linear viscoelastic fluid model over a stretch$\mathrm{ing} /$ shrinking sheet in the region of stagnation point flows", Scientia Iranica, 19(6), pp. 1541-1549 (2012).

7. Eswaramoorthi, S., Bhuvaneswari, M., Sivasankaran, S., and Rajan, S. "Effect of radiation on MHD convective flow and heat transfer of a viscoelastic fluid over a stretching surface", Procedia Eng., 127, pp. 916-923 (2015).

8. Mishra, S.R., Pattnaik, P.K., Bhatti, M.M. and Abbas, T. "Analysis of heat and mass transfer with MHD and chemical reaction effects on viscoelastic fluid over a stretching sheet", Indian J. Phys., 91(10), pp. 1219-1227 (2017).

9. Lee, J., Kandaswamy, P., Bhuvaneswari, M. and Sivasankaran, S. "Lie group analysis of radiation natural convection heat transfer past an inclined porous surface", J. Mech. Sci. Tech., 22(9), pp. 17791784 (2008).
10. Beg, O.A., Uddin, M.J., Rashidi, M.M., and Kavyani, N. "Double-diffusive radiative magnetic mixed convective slip flow with Biot and Richardson number effects", J. Engin. Thermophys., 23(2), pp. 79-97 (2014).

11. Ellahi, R., Bhatti, M.M., and Vafai, K. "Effects of heat and mass transfer on peristaltic flow in a nonuniform rectangular duct", Int. J. Heat Mass Transf., 71, pp. 706-719 (2014).

12. Niranjan, H., Sivasankaran, S., and Bhuvaneswari, M. "Analytical and numerical study on magnetoconvection stagnation-point flow in a porous medium with chemical reaction, radiation, and slip effects", Mathe. Prob. Eng., Article ID 4017076, pp. 1-12 (2016).

13. Sheikholeslami, M. and Rashidi, M.M. "Non-uniform magnetic field effect on nanofluid hydrothermal treatment considering Brownian motion and thermophoresis effects", J. Braz. Soc. Mech. Sci. Eng., 38(4), pp. 1171-1184 (2016).

14. Sheikholeslami, M. and Bhatti, M.M. "Forced convection of nanofluid in presence of constant magnetic field considering shape effects of nanoparticles", Int. J. Heat Mass Transf., 111, pp. 1039-1049 (2017).

15. Chamkha, A.J. "Hydromagnetic three-dimensional free convection on a vertical stretching surface with heat generation or absorption", Int. J. Heat Fluid Flow, 20(1), pp. 84-92 (1999).

16. Patil, P.M., Roy, S., and Chamkha, A.J. "Double diffusive mixed convection flow over a moving vertical plate in the presence of internal heat generation and a chemical reaction", Turkish J. Eng. Envir. Sci., 33, pp. 193-205 (2009).

17. Bhuvaneswari, M., Sivasankaran, S., and Kim, Y.J. "Lie group analysis of radiation natural convection flow over an inclined surface in a porous medium with internal heat generation", J. Porous Media, 15(12), pp. 1155-1164 (2012).

18. Mukhopadhyay, S. and Layek, G.C. "Effects of variable fluid viscosity on flow past a heated stretching sheet embedded in a porous medium in presence of heat source/sink", Meccanica, 47(4), pp. 863-876 (2012).

19. Ganga, B., Saranya, S., Vishnu Ganesh, N., and Abdul Hakeem, A.K. "Effects of space and temperature dependent internal heat generation/absorption on MHD flow of a nanofluid over a stretching sheet", J. Hydrodyn., 27(6), pp. 945-954 (2015).

20. Kasmani, R.M., Sivasankaran, S., Bhuvaneswari, M., and Siri, Z. "Effect of chemical reaction on convective heat transfer of boundary layer flow in nanofluid over a wedge with heat generation/absorption and suction", J. Appl. Fluid Mech., 9(1), pp. 379-388 (2016).

21. Karthikeyan, S., Bhuvaneswari, M., Sivasankaran, S., and Rajan, S. "Soret and Dufour effects on MHD mixed convection heat and mass transfer of a stagnation point flow towards a vertical plate in 
a porous medium with chemical reaction, radiation and heat generation", J. Appl. Fluid Mech., 9(3), pp. 1447-1455 (2016).

22. Hayat, T., Shehzad, S.A., Qasim, M., and Obaidat, S. "Flow of a second grade fluid with convective boundary conditions", Ther. Sci., 15(2), pp. S253S261 (2011).

23. Makinde, O.D. "Similarity solution for natural convection from a moving vertical plate with internal heat generation and a convective boundary condition", Ther. Sci., 15(1), pp. S137-S143 (2011).

24. Shehzad, S.A., Alsaedi, A., and Hayat, T. "Three dimensional flow of Jeffery fluid with convective surface boundary conditions", Int. J. Heat Mass Transf., 55(15-16), pp. 3971-3976 (2012).

25. Merkin, J.H. and Pop, I. "The forced convection flow of a uniform stream over a flat surface with a convective surface boundary condition", Commun. Nonlinear Sci. Numer. Simulat., 16(9), pp. 3602-3609 (2011).

26. Eswaramoorthi, S., Bhuvaneswari, M., Sivasankaran, S., and Rajan, S. "Soret and Dufour effects on viscoelastic boundary layer flow, heat and mass transfer in a stretching surface with convective boundary condition in the presence of radiation and chemical reaction", Scientia Iranica $B, \mathbf{2 3}(6)$, pp. 2575-86 (2016).

\section{Biographies}

Marimuthu Bhuvaneswari received MSc, MPhil, and $\mathrm{PhD}$ degrees from Bharathiar University, Coimbatore, India. She received Post-Doctoral Fellowship twice from National Cheng Kung University, Taiwan, and twice from Sungkyunkwan University, South Korea. Then, she worked as a research fellow at University of Malaya, Malaysia. Her areas of interest are numerical and analytical methods for PDE and radiation, heat, and mass transfer.
Sheniyappan Eswaramoorthi is an Assistant Professor in the Department of Mathematics at Dr. N. G. P. Arts and Science College, Coimbatore, Tamilnadu, India. He obtained his MSc, MPhil, and PhD degrees from Bharathiar University, Coimbatore, India. His areas of interest are boundary layer flow, and heat and mass transfer.

Sivanandam Sivasankaran received his MSc, MPhil, and $\mathrm{PhD}$ degrees from Bharathiar University, India. Then, he received Post-Doctoral Fellowship from National Cheng Kung University and National Taiwan University, Taiwan. He was a Research Professor at Yonsei University, South Korea. Also, he was an Assistant Professor at Sungkyunkwan University, South Korea, and Senior Lecturer at University of Malaya, Malaysia. Presently, he works at King Abdulaziz University, Saudi Arabia. He is an Associate Editor of two journals and a member of the editorial boards of several international journals. His areas of interest are convective heat and mass transfer, CFD, nanofluids, micro-channel heat sinks, and porous media.

Subbarayagounder Rajan is an Associate Professor and the Head of Mathematics at Erode Arts and Science College, Erode, Tamilnadu, India, having 25 years of teaching experience at both undergraduate and postgraduate levels. He received his MSc in 1984 from Madras University, and MPhil in 1995 and PhD in 2005 from Bharathiar University, India. His areas of interest are computer algebra systems and applications of differential equations.

Ali Saleh Alshomrani is an Assistant Professor of Mathematics at King Abdulaziz University, Saudi Arabia. He received $\mathrm{MSc}$ and $\mathrm{PhD}$ degrees from Heriot Watt University, Edinburgh, United Kingdom. His areas of interest are numerical methods, dynamical systems, and applied analysis. 